\title{
A type 2 diabetes mellitus prevention index predicts incident diabetes in the UK Women's Cohort Study
}

\author{
L. Ashton, J. E. Cade and V. J. Burley \\ School of Food Science \& Nutrition, University of Leeds, Leeds LS2 9JT, UK
}

Worldwide approximately 366 million people had diabetes in 2011, with around $90 \%$ constituting type 2 diabetes mellitus (T2DM); by 2030 this will have risen to 552 million unless appropriate prevention strategies are implemented (IDF 2012). The aim of this study was to create a dietary type 2 diabetes prevention index score (T2DPIS) and assess the association of the score with incidence of T2DM in the UK Women's Cohort Study (UKWCS).

The UK Women Cohort's Study is a population-based prospective cohort study of middle-aged women. From 35,372 women who completed a 217-item food frequency questionnaire (FFQ) between 1996 and 1999, 12,149 women also completed a follow-up questionnaire after an average of 4 years. At each of these contacts, women reported whether their doctor had diagnosed them with diabetes. After excluding prevalent cases, and those with unfeasible energy intakes, 114 women reported having developed diabetes during the follow-up period and were therefore included in the analysis as incident cases. The remaining 11,982 women who remained free from diabetes were defined as non-cases. The T2DPIS was based on frequency responses from the baseline FFQ for 11 main food groups for which there was prior literature evidence of an association with incidence of T2DM. Reverse scoring was applied to food groups which were linked with increased risk of T2DM. The maximum score possible was 100 and the resulting scores for the UKWCS participants ranged from 19 to 83. We hypothesized that women achieving a high ('healthier') score using this index were therefore potentially consuming a diet that might be associated with a lower odds of developing T2DM during the follow-up period. Logistic regression analysis was used to determine the relationship between the T2DPIS and odds of being an incident case of T2DM. Two models were run, an age-adjusted and a multivariable-adjusted model. Results in the table are expressed as the odds of developing incident T2DM for each 10 unit increment of the T2DPIS and also for each score quintile.

Women in the highest quintile for the T2DPIS (those with the 'healthiest' dietary pattern) were at decreased odds of T2DM when compared with those in the lowest quintile. The OR for the age-adjusted model was 0.48 (95\% CI; 0.27, 0.86, $p$ trend $=0.01)$. Adjustment for a range of covariates did not modify this association: $0.49(95 \%$ CI $0.24,0.98 \mathrm{p}$ trend $=0.02)$ and further inclusion of BMI and energy intake in the model did not materially alter the point estimates (data not shown).

\begin{tabular}{|c|c|c|c|c|c|c|c|c|}
\hline \multirow[b]{2}{*}{ Model } & \multirow[b]{2}{*}{ Cases/non-cases } & \multicolumn{5}{|c|}{ Type 2 Diabetes Prevention Index Score } & \multirow[b]{2}{*}{ Continuous $\mathrm{OR}^{\neq}$} & \multirow[b]{2}{*}{$\mathrm{P}_{\text {trend }}$} \\
\hline & & Q1 referent & Q2 & Q3 & Q4 & Q5 & & \\
\hline Age adjusted & $113 / 11919$ & 1 & $0.66(0.38,1.15)$ & $0.60(0.35,1.02)$ & $0.44(0.25,0.78)$ & $0.48(0.27,0.86)$ & $0.73(0.57,0.93)$ & 0.01 \\
\hline Multi-variable* & $88 / 10390$ & 1 & $0.90(0.47,1.70)$ & $0.73(0.38,0.37)$ & $0.51(0.26,0.99)$ & $0.49(0.24,0.99)$ & $0.71(0.53,0.95)$ & 0.02 \\
\hline
\end{tabular}

Values are odds ratios (95\% CI's) calculated from logistic regression. *Adjusted for age, smoking, ethnicity, physical activity, family history of diabetes, family history of

hypertension, family history of hypercholesterolemia. $\neq$ Odds ratio of being a case for each 10 -unit score increment.

Following a healthy dietary pattern as characterized by the type 2 diabetes prevention index score was associated with a $50 \%$ reduction in odds of developing diabetes in participants of the UKWCS. Consequently, this index has potential for use in public health settings as a dietary guide for prevention of type 2 diabetes.

International Diabetes Federation (2012) IDF diabetes atlas. [online]. [Accessed 03 April 2013]. Available from: http://www.diabetesatlas.org 